\title{
Flow Control Using Passive Vortex Generators
}

\author{
Suyash Tandon, Siddhesh Shinde†, Kevin Maki and Eric Johnsen ${ }^{\S}$ \\ University of Michigan, Ann Arbor, MI, 48109, USA
}

\begin{abstract}
Passive vortex generators (VGs) can be employed to control boundary layer separation effectively. As the boundary layer encounters an adverse pressure gradient, it tends to separate from the surface due to a momentum deficit. Passive VGs entrain high momentum fluid from the mean flow and enhance mixing which energizes the near-wall flow. In this study the performance of a single cube used as a passive VG to control flow separation on a backward-facing ramp is evaluated using wall-resolved large-eddy simulations. The Reynolds number of the flow is 19,600. Since our interests lie in low-profile VGs within a turbulent boundary layer, we evaluate cube height to boundary layer thickness ratio of 0.6 to understand the effect of the physical VG on the separation region.
\end{abstract}

\section{Introduction}

Despite significant efforts dedicated to understanding flow separation, implementation of this knowledge for flow control is not fully straight forward. Manipulation of wall-bounded flows using active and passive approaches is an active area of research. The ability to control boundary layer separation effectively has been shown to reduce drag and increase lift on airfoils at moderate Reynolds number, ${ }^{1}$ to reduce losses in subsonic diffusers ${ }^{2}$ and to reduce afterbody drag in aircraft fuselages. ${ }^{3}$

Due to large losses of energy associated with boundary layer separation in a variety of applications it is important to be able to control boundary layer separation. One possible approach is to energize the boundary layer by entraining momentum from the free stream to the near wall region by increasing turbulent mixing. For a fixed separation point, solid obstacles i.e., passive VGs, placed inside the boundary layer may achieve this goal. Extensive research has been done in passive flow control and VG geometries such as cylinders, the wheeler doublets or wish-bones have been studied. Traditionally, VGs whose characteristic size $h$ is on the order of the boundary layer thickness $\delta$ have been used in aerospace applications for localized flow control over short streamwise distances. ${ }^{4}$ However, these VGs have a greater device drag associated with them on account of their large size. A better-suited VG configuration can be designed by using low-profile VGs for applications where the separation point is fixed. An in depth review of investigations using submerged VGs for boundary layer separation control is given in Lin. ${ }^{4}$ This exploratory study suggests that the submerged VGs with $h / \delta \leqslant 0.65$ have better performance because of the low device drag. Studies by $\operatorname{Lin}^{4}$ and Rao and Kariya ${ }^{5}$ show that the effectiveness in controlling the boundary layer separation by employing a VG depends on the size of the VG relative to the boundary layer thickness, the spanwise spacing between the VGs and the streamwise distance between the VG trailing edge and the line of separation.

Prior studies on passive flow control ${ }^{4}$ have been primarily experimental. Early computational studies have used Reynolds-Averaged Navier-Stokes (RANS) approach ${ }^{6}$ with a constant time-averaged velocity inlet boundary condition. However, such approaches do not adequately represent separation of spatially evolving turbulent boundary layers such as on the aft of a blunt object. The goal of the present study is to use wallresolved Large Eddy Simulation (LES) to numerically investigate the role of submerged cubic VGs on flow

\footnotetext{
${ }^{*}$ Graduate Student, Department of Mechanical Engineering, 1231 Beal Ave., 2043 Walter E. Lay Automotive Laboratory, Ann Arbor, MI 48109-2133, AIAA member.

${ }^{\dagger}$ Graduate Student, Department of Mechanical Engineering, 1231 Beal Ave., 2043 Walter E. Lay Automotive Laboratory, Ann Arbor, MI 48109-2133, AIAA member.

${ }^{\ddagger}$ Assistant Professor, Naval Architecture and Marine Engineering, 210 NAME Building, 2600 Draper Drive, Ann Arbor, MI 48109-2145, AIAA member.

$\S$ Assistant Professor, Department of Mechanical Engineering, 1231 Beal Ave., 2043 Walter E. Lay Automotive Laboratory, Ann Arbor, MI 48109-2133, AIAA Senior member.
} 
separation of a spatially evolving turbulent boundary layer on a $25^{\circ}$ backward-facing ramp. This canonical geometry is relevant to many applications such as expansion nozzles, the rear end of a blunt object, while cubes reduce the number of characteristic lengths associated with the VGs. Wall resolved LES will help capture the detailed time-dependent flow features necessary to develop flow control strategies. The paper is organized as followed. After describing the governing equations and computational approach in section II, the problem set-up is presented in section III. Results are discussed in detail in section IV, before concluding.

\section{Governing Equations}

We solve the incompressible Navier-Stokes equations for velocity $\mathbf{u}$ and pressure $p$ :

$$
\begin{gathered}
\mathbf{u}_{t}+\mathbf{u} \cdot \nabla \mathbf{u}-\nu \Delta \mathbf{u}+\frac{1}{\rho} \nabla p=\mathbf{f} \quad \text { in } \Omega \times(0, \mathrm{~T}), \\
\nabla \cdot \mathbf{u}=0 \quad \text { in } \Omega \times(0, \mathrm{~T}) .
\end{gathered}
$$

where $\nu$ is the kinematic viscosity, $\rho$ is the density, $\mathbf{f}$ is the body force, and $\Omega \subset \mathbb{R}^{3}$ is the bounded flow domain with a sufficiently regular boundary $\partial \Omega$. The equations are applied with the initial conditions

$$
\mathbf{u}=\mathbf{u}_{0}(\mathbf{x})
$$

and appropriate boundary conditions, such as the no-slip condition,

$$
\mathbf{u}=\mathbf{0} \quad \text { on } \quad \partial \Omega .
$$

For our numerical investigation we model the NSE $(1,2)$ using a second-order explicit central discretization scheme in space and second-order implicit time marching method in an unstructured finite volume method, built in OpenFOAM®.

\section{II.A. Numerical setup}

The filtered NSE in $\mathrm{LES}^{7}$ are solved using the dynamic one-equation eddy-viscosity model ${ }^{8}$ in OpenFOAM@. A second-order implicit time marching scheme is used along with second-order central scheme for the convective term and second-order central scheme with explicit correction for the diffusion term. The simulations are carried out for a total time period of $1500 \mathrm{H} / U_{\infty}$ with variable time steps to achieve a maximum Courant number of 1 . When needed, statistical averaging is performed from $t=375 H / U_{\infty}$. We allow 50 "flow throughs" before statistically averaging the data to avoid contamination of results due to the initial transient. ${ }^{9}$ The time required for one "flow through" is defined as time required for the flow to move past the downstream surface of the ramp with an average velocity, $U_{\text {avg }} \approx 0.8 U_{\infty}$.

\section{Computational setup}

\section{III.A. Geometry}

As shown in Figure 1, the geometry consists of a backward-facing ramp of height $H=2.4 \mathrm{~cm}$ and angle $25^{\circ}$. A prescribed turbulent boundary layer ${ }^{9}$ enters the domain with $\delta_{o}=0.416 H$. The Reynolds number based on the free-stream velocity and ramp height is 19,600. This boundary layer takes $12 \delta_{0}$ lengths in the streamwise direction to recover the prescribed thickness at the inlet. ${ }^{9,10}$ Therefore in order to achieve the thickness of the boundary layer at the cube location similar to the prescribed thickness at the inlet, the upstream part of the domain before the ramp edge is is $12 \delta_{o}+4 h$ long. Zero-gradient conditions are applied at the domain's end. Based on past investigations pertaining to the dependence of the flow physics on the domain size, the domain is $10 h$ wide, with slip-wall boundary conditions in the spanwise direction. The domain is $6 \mathrm{H}$ tall with no slip-wall boundary conditions along the bottom wall of the domain while slip-wall boundary conditions are prescribed on the top wall of the domain. The domain dimensions are specified in Table 1.

A single cubic vortex generator is placed a distance $x_{v g}$ upstream of the ramp edge, aligned with the centerplane of the domain. In the present study, we hold $x_{v g}=3$ as a constant and consider the following scaled cube heights: $h / \delta_{o}=0.6$. As a baseline, we also simulate the problem with no VG. 


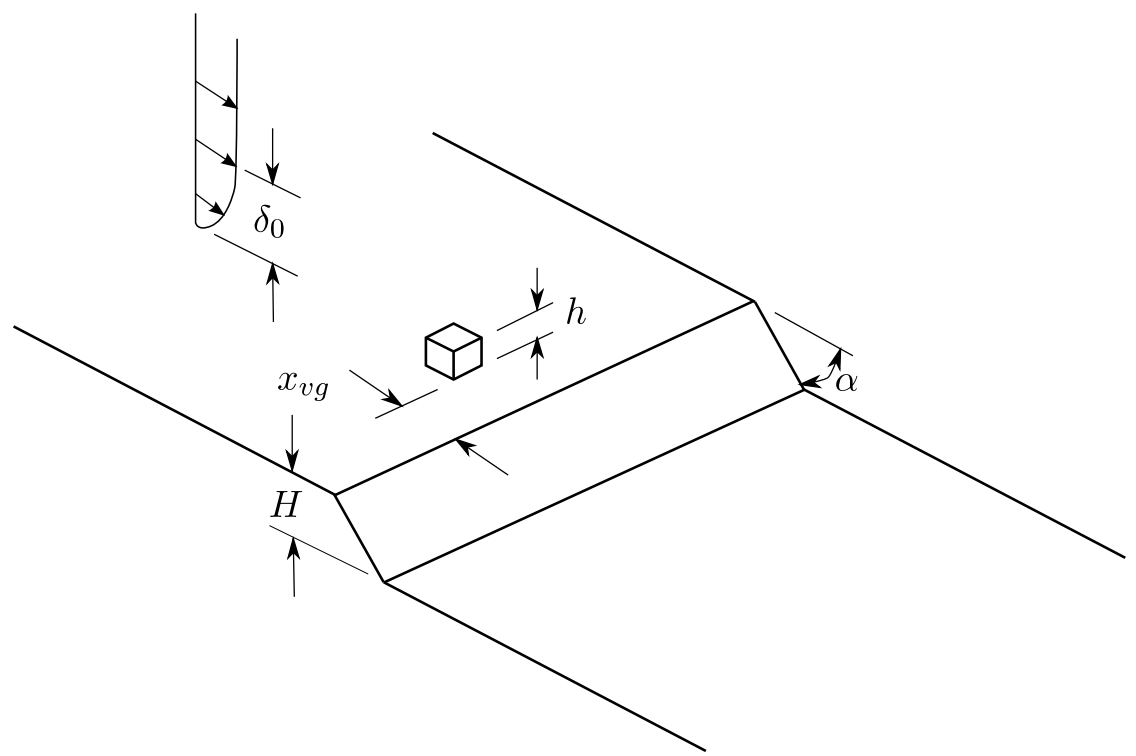

Figure 1. Schematic of the flow domain.

Table 1. Domain specification

\begin{tabular}{lc} 
Parameter & Dimension \\
\hline Upstream Plate Length & $12 \delta_{0}+4 h$ \\
Downstream Plate Length & $6 H$ \\
Spanwise Length & $10 h$
\end{tabular}

\section{III.B. Mesh specification}

As shown in Tables 2 and 3, we use a sequence of meshes ranging from coarse to fine. To ensure appropriate resolution at the walls, the average grid spacing in wall coordinates is $\Delta_{y}^{+}<1$ in the wall-normal direction while a uniform resolution with $\Delta_{x}^{+}, \Delta_{z}^{+} \approx 25$. Computational cost is reduced by mapping the results from coarser mesh onto the successive finer mesh as an initial condition. The coarser grid had around 19.29 million cells, while the finest mesh had 27.16 million cells.

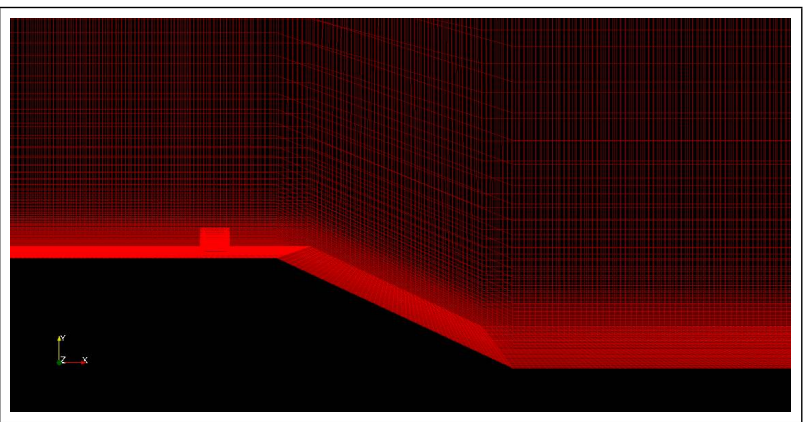

(a) Front view.

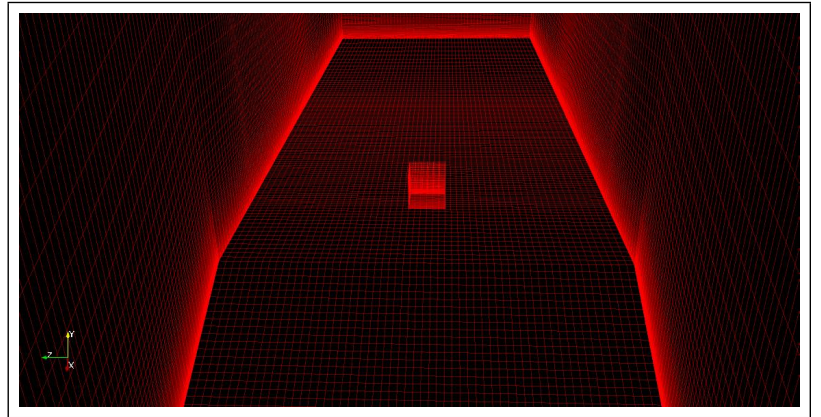

(b) Side view looking towards the inlet.

Figure 2. Low profile cube, $h / \delta_{0}=0.6$ case mesh with local refinement near the cube and ramp.

We locally refine the mesh such that the resolution is nearly uniform from $3 H$ upstream of the ramp edge to $5 H$ downstream of the ramp as shown in Figure 2. In the wall-normal direction, the grid spacing is uniform upto $\Delta_{y}^{+}=50$. The vortical structures around the cube are not affected by the domain width $(W)$ 
Table 2. Meshing Information for the baseline problem with no VG.

\begin{tabular}{lccc} 
Property & Coarse mesh & Medium mesh & Fine mesh \\
\hline Total number of cells $\left(\times 10^{6}\right)$ & 17.13 & 26.26 & 31.28 \\
$\Delta^{+*}$ in the refined region & 5.0 & 1.0 & 0.8
\end{tabular}

${ }^{*}$ The "+" indicates the dimensionless grid spacing in wall coordinates. $\Delta^{+}=\frac{\Delta u_{\tau}}{\nu}$ where $\Delta$ is the grid spacing in physical dimensions, $u_{\tau}$ is the friction velocity at the wall and $\nu$ is the kinematic viscosity of the fluid.

Table 3. Meshing information for the problem with a $h / \delta_{0}=0.6 \mathrm{VG}$.

\begin{tabular}{lccc} 
Property & Coarse mesh & Medium mesh & Fine mesh \\
\hline Total number of cells $\left(\times 10^{6}\right)$ & 19.29 & 23.21 & 27.16 \\
Number of cells $/ h$ & 60 & 100 & 120 \\
$\Delta^{+*}$ in the refined region & 5.0 & 1.0 & 0.8
\end{tabular}

as long as $W / h>5 .{ }^{11}$ From the LES results of flow past a cube placed in a channel ${ }^{6}$ it was found that the horse-shoe vortex formed in front of the cube extends to a distance of $1.6 \mathrm{~h}$ upstream of the cube and that the flow is quasi-potential for $y / H>2$.

\section{Results and discussion}

\section{IV.A. Comparison between the $\mathrm{h} / \delta_{0}=0.6$ case and the ramp with no VG}

To understand the disturbing effect of a VG, we compare the flow fields for the problem under consideration with no VG to that with a VG of scaled height $h / \delta_{o}=0.6$. Figure $3(\mathrm{a})-3(\mathrm{~b})$ shows streamlines overlayed on the speed contours corresponding to these two cases.

When there is no VG, the presence of a surface discontinuity at the ramp edge imposes a pressure gradient in the streamwise direction and causes the flow to separate at the edge of the ramp as seen in Figure 3(a) and the flow to reverse it's direction, characterized by a recirculation region extending several $H$ downstream. The flow reattaches eventually on the bottom surface. When the VG is present, the vortical structures emanating from the flow around the cube mitigate flow separation by energizing the boundary layer. ${ }^{9}$ As seen from Figure 3(b) the flow separates on top of the cube and forms a recirculating bubble in the cube's wake. The flow reattaches behind the cube before reaching the upper edge of the ramp. Instead of the large recirculation region along the ramp when no VG is present, two small recirculation regions are observed along the ramp. The total size of the separated region is smaller when a vortex generator is present.

To better understand the behavior of the flow in the separation region and the effect of VG, we sample the mean streamwise velocity profiles along the center plane at different streamwise locations.

The mean streamwise velocity profiles shown in Figure 4(a) - 4(d) are sampled on the upstream plate for both with and no VG cases. At inlet velocity profiles in Figure 4(a) are completely attached to the bottom wall located at $y / H=1$. At the location of the front face of the cube, in Figure 4(b), we can clearly see that in the case without a VG the boundary layer is completely attached to the surface whereas a region with velocity defect can be observed in cases with the VG due to the presence of the front face of the VG. The smaller spikes on top of the front face of the VG are attributed to the complex flow features originating from the separation along the top edge. Similar flow recirculation can be seen at the edge on back face of the VG while the flow on top accelerates. The flow in the case of without a VG remains attached to the bottom wall as seen in Figure 4(c). In the case without a VG the flow separates at the ramp edge; the flow accelerates while the boundary layer thickens. The absence of negative velocity in the presence of a VG as in Figure 4(d) suggests that the flow in the wake of VG in the center plane is attached to the bottom wall.

Figure 5(a) - 5(d) shows the mean streamwise velocity profiles sampled along the center plane at different locations on the ramp surface and the downstream plate, thus providing a more quantitative measure of the flow disruption due to the VG. At $x / H=1$, in Figure 5(a), the negative flow in the presence of a VG is lower in magnitude as compared to the case without VG and is restricted closer to the wall. As we move 


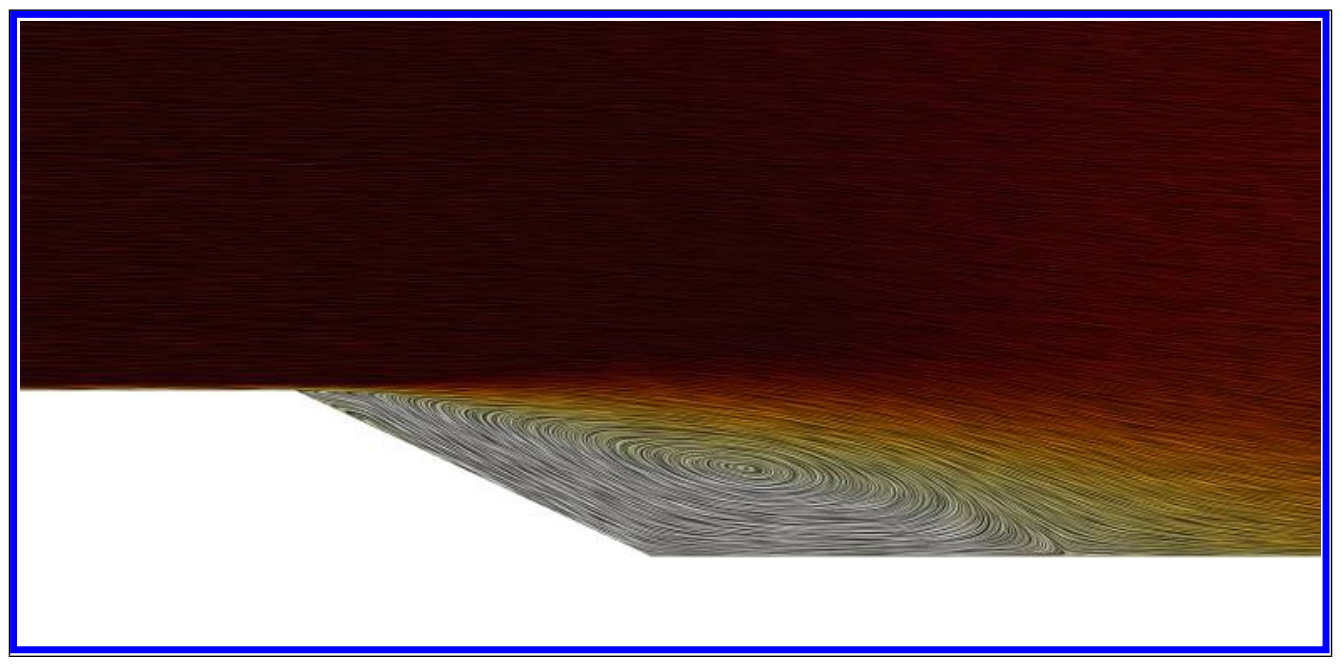

(a) Without VG.

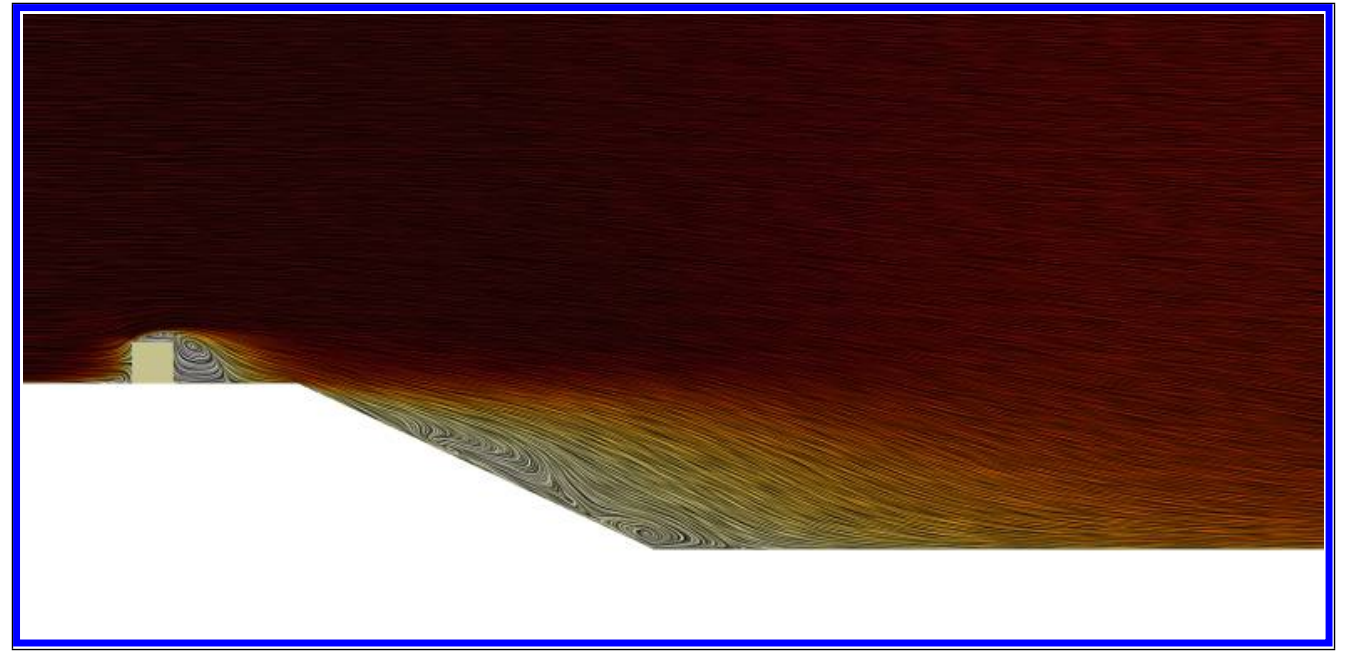

(b) With VG.

Figure 3. Line Integral Convolution (LIC) image of mean streamwise velocity streamlines in the plane of symmetry.

downstream to the bottom edge of the ramp at $x / H=2$ we see a that the flow with no VG has a large separation region that extends further above the bottom wall located at $y / H=0$. The flow with VG has a smaller region of recirculation shown in Figure 5(b). The velocity profiles in Figure 5(c) sampled at $x / H=4$ suggests that the flow in case of VG is attached to the bottom wall as evidenced by the absence of negative velocity which means that flow attaches between the bottom edge of the ramp at $x / H=2$ and $x / H=4$. This behavior changes at $x / H=5$ for both cases with and without VG where the flow is attached to the bottom wall.

To identify the point of separation and reattachment we consider the time-averaged skin friction coefficient and the coefficient of surface pressure along the bottom wall in the plane of symmetry. In the case with no VG in Figure $6(\mathrm{a})$ at $x / H=0$ the sharp drop in the skin friction marks the point of flow separation along ramp top edge. The skin friction is negative from $x / H=0$ to 2.1 because of the recirculation bubble on the ramp. The sharp spike at $x / H=2.1$ is due to near stagnation conditions at the bottom edge of the ramp. Along the bottom wall of the ramp between $x / H=2.1$ and 3.0 the negative velocity close to the wall increases followed by a recovery of the velocity to a positive value near the wall at $x / H=4.8$. Beyond this point the boundary layer attaches to the bottom wall and it begins to recover a conventional turbulent boundary layer structure. Similar observations can be made from Figure 6(b). The wall pressure coefficient shows a favorable pressure gradient on the surface upstream of the ramp followed by a region of uniform value on the ramp where the flow is separated. At $x / H=2.1$ we observe a pressure spike due to the 


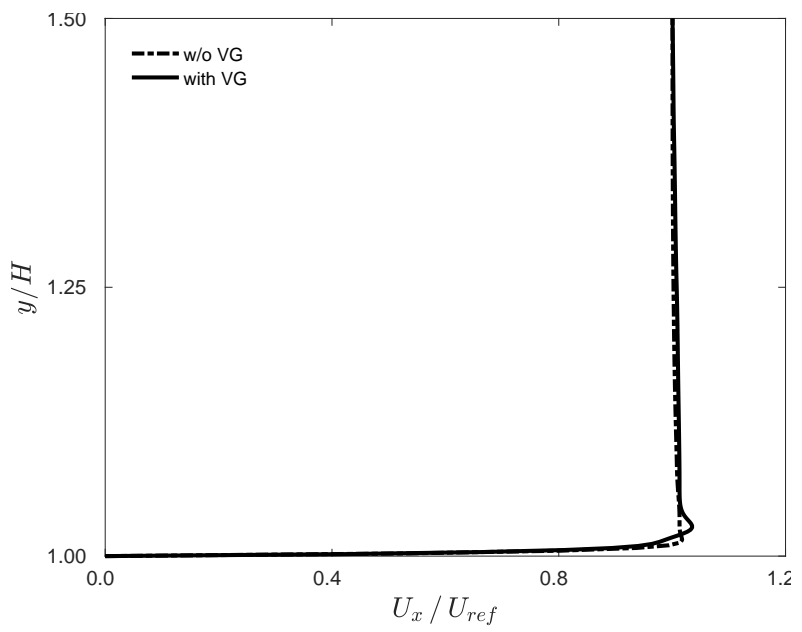

(a) At inlet.

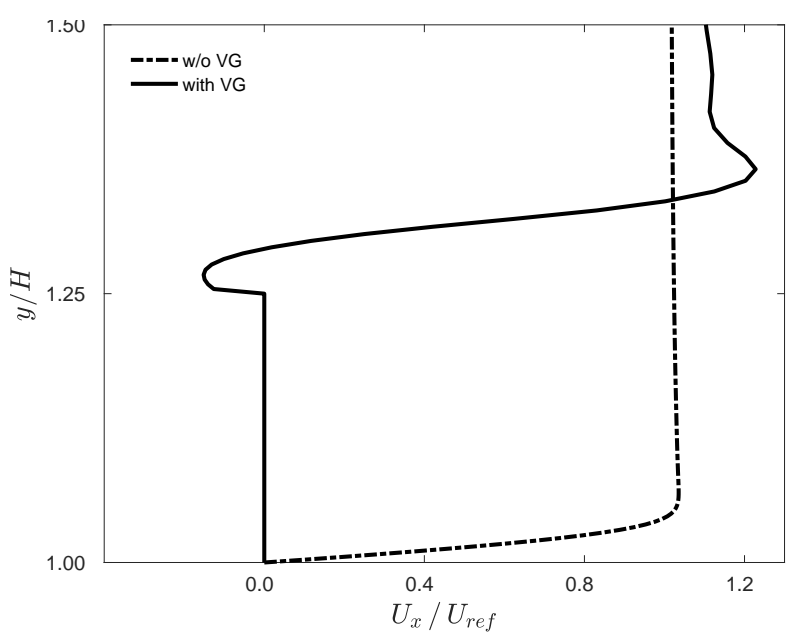

(c) At back face of VG.

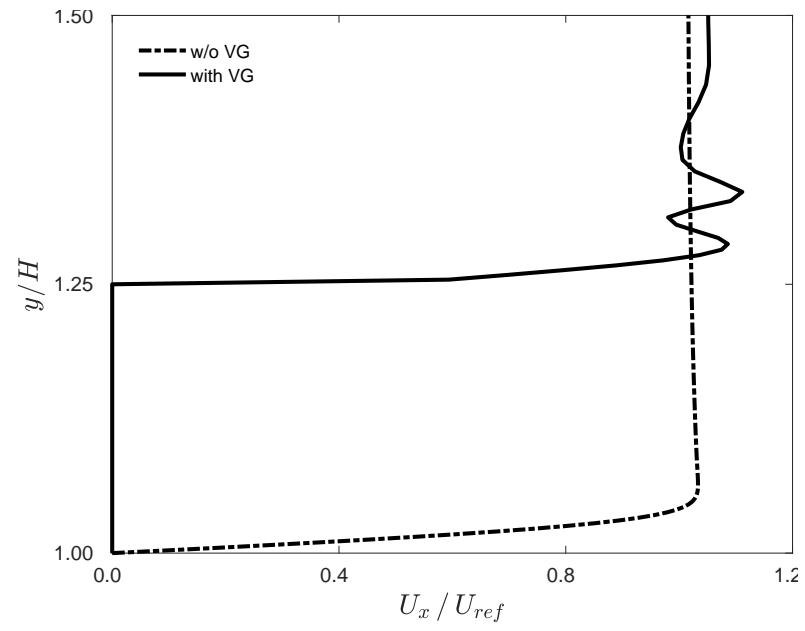

(b) At front face of VG.

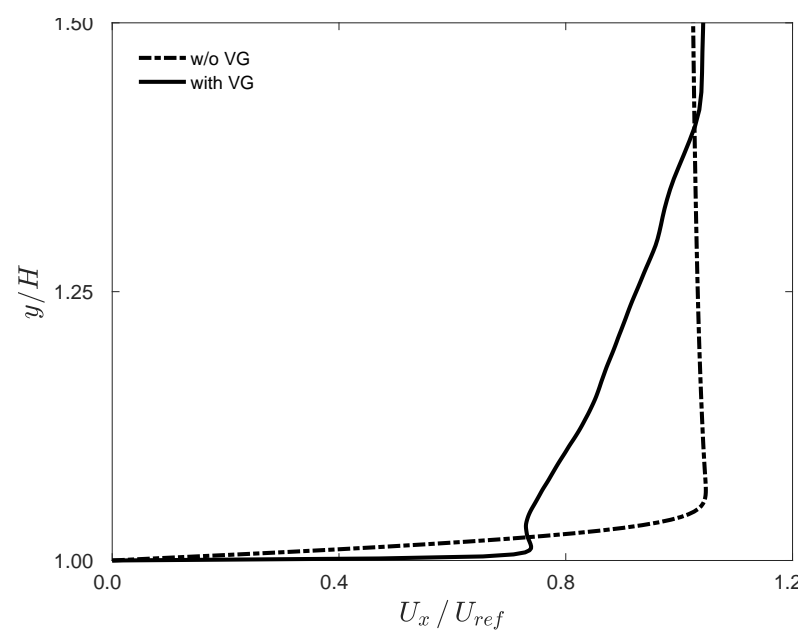

(d) At upper ramp edge.

Figure 4. Streamwise component of the mean velocity profile sampled at different streamwise locations along the center plane on the upstream plate for simulations without a VG and with a physical VG.

stagnation conditions at the bottom ramp edge. The flow gets attached to the bottom wall around $x / H=5$. This point is marked by a positive $C_{f}$ in Figure 6(a) a favorable pressure gradient.

In the presence of the VG at $x / H=-0.75$ a large fluctuation in $C_{f}$ and $C_{p}$ are noticeable in the vicinity of the cube from Figures 6(a) - 6(b). The stagnation of flow in front of the cube and recirculation near the back of the cube causes negative skin friction. It also leads to high stagnation pressure in front of the cube and a low pressure region near the back surface. On the ramp surface the flow is attached initially as suggested by a positive $C_{f}$ and constant wall pressure distribution before it separates again halfway down the ramp. Near the bottom ramp corner a small value of negative skin friction suggests reverse flow, but it has a smaller velocity magnitude compared to the case with no VG. The flow attaches to the surface at $x / H=2.8$. The attachment length is reduced by $2 H$ with the use a passive VG.

In the absence of a VG, the turbulent kinetic energy, TKE is produced from the shear layer emanating from the ramp edge after the flow separates as seen from Figure 7(a). The turbulent kinetic energy magnitude near the wall on the ramp and downstream plane is small. On the other hand, the presence of a VG generates intense turbulent kinetic energy in the vicinity of the VG, i.e., before the flow separates from the ramp edge. This timely production of TKE by the VG energizes the flow and reattaches it earlier. The peak TKE is observed along the edges of the VG near the front face and is subsequently dispersed in the wake of the cube, see Figure 7(b). The perturbed flow by the VG manipulates the vorticity distribution in the wake of 


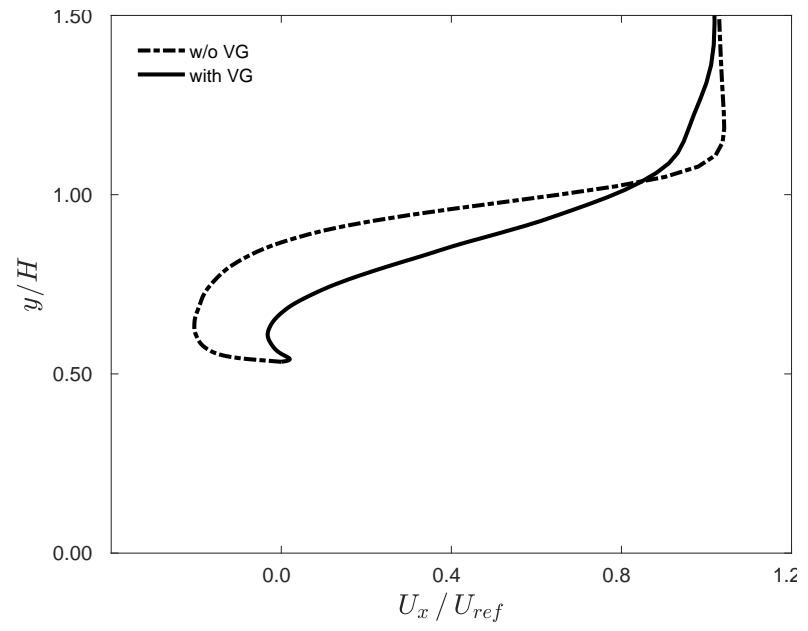

(a) At $x / H=1$.

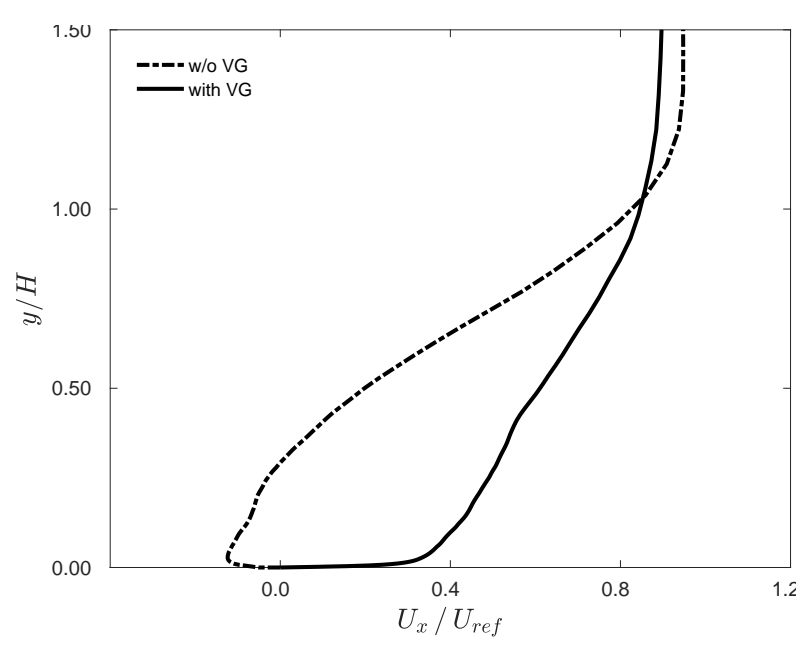

(c) At $x / H=4$.

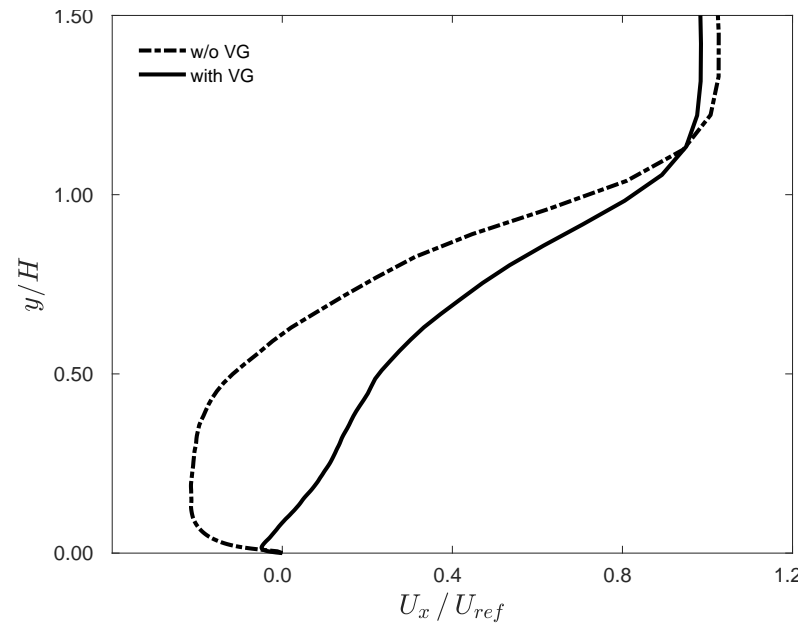

(b) At $x / H=2$.

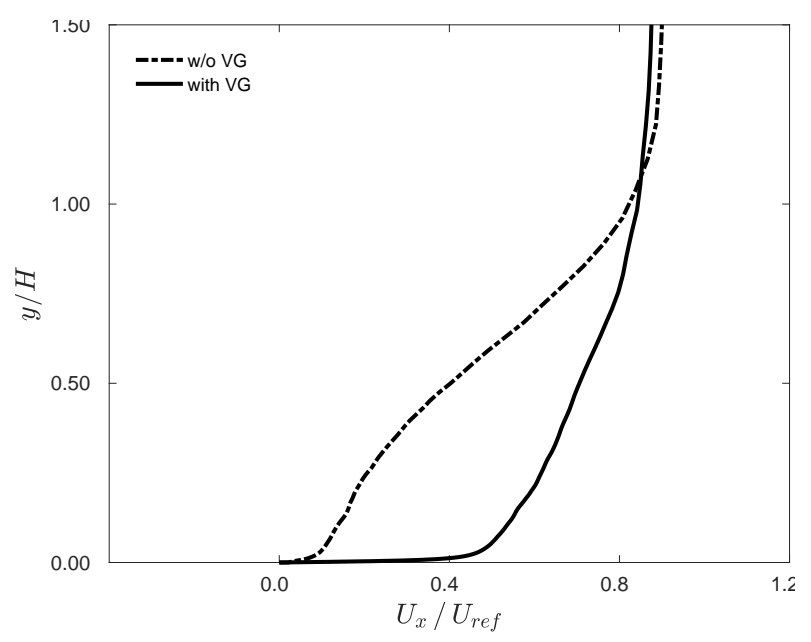

(d) At $x / H=5$.

Figure 5. Streamwise component of the mean velocity profile sampled at different streamwise locations along the center plane on the ramp surface and downstream plate for simulations without a VG and with a physical VG.

the VG which energizes the flow in the vicinity of the bottom wall. This energy is carried downstream along with the shed vortices due to which the magnitude of TKE is more near the bottom wall in the downstream section of the model.

\section{Conclusions and Future Work}

We numerically investigated the role of a single submerged cubic vortex generator placed upstream of a 25-degree ramp using wall-resolved LES. We observe that the presence of the cube reduces the size of the separated flow region. The VG produces intense turbulent kinetic energy in its vicinity, upstream from the ramp. We attribute the reduction in separation to this energizing of the boundary layer. The skin friction coefficient and wall pressure distribution along the plane of symmetry provide evidence that the flow has a smaller region of recirculation near the bottom ramp edge and the reattachment length is reduced by two ramp height lenths when a single VG is used. 


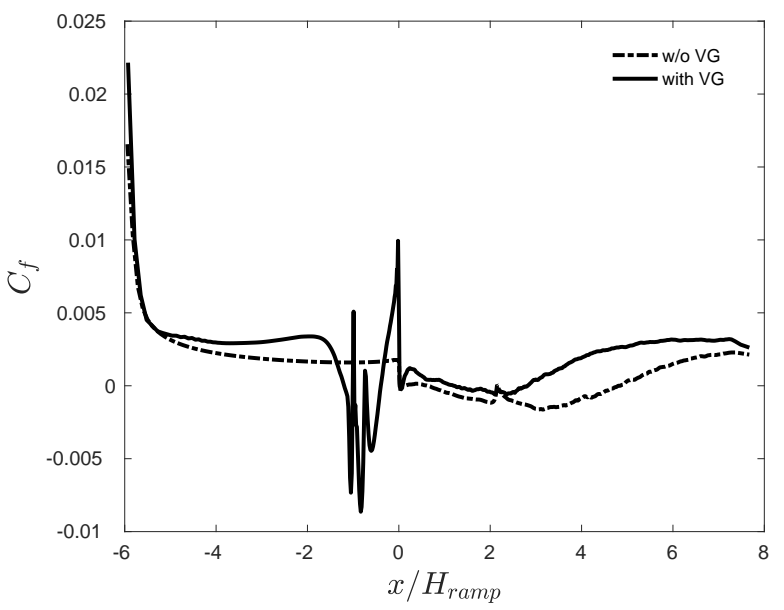

(a) Skin friction coefficient.

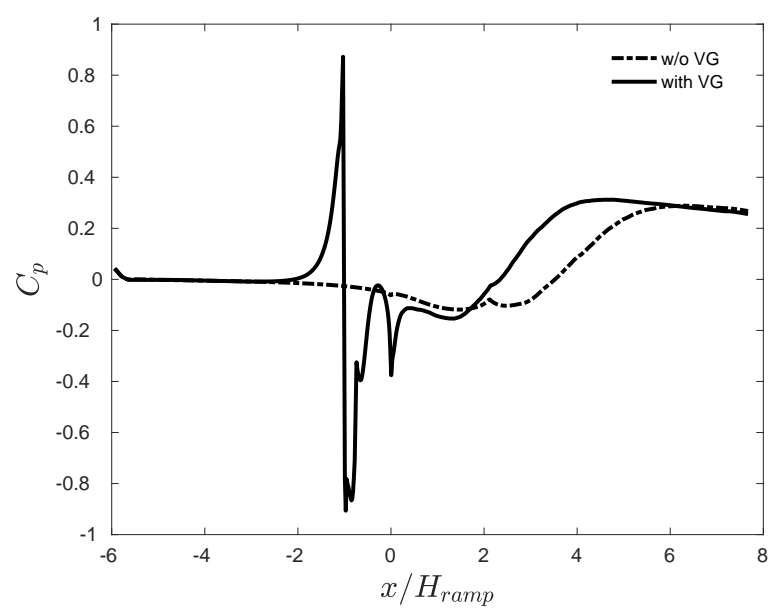

(b) Wall pressure coefficient.

Figure 6. Skin friction and wall pressure coefficient along the bottomwall in the plane of symmetry.

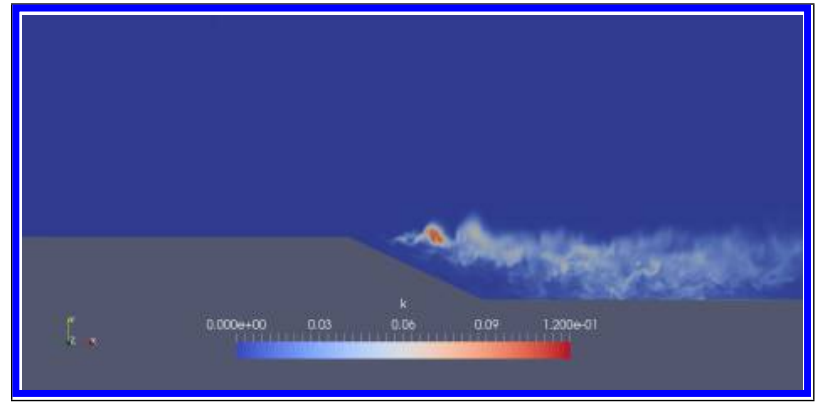

(a) Without VG.

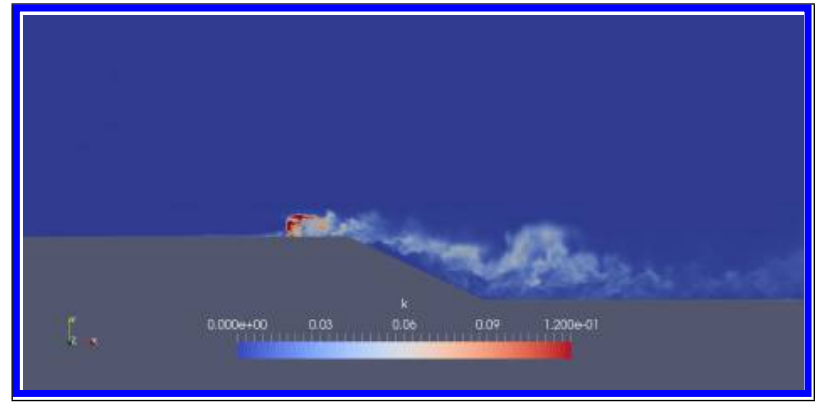

(b) With VG.

Figure 7. Turbulent kinetic energy contours along the center plane.

\section{Acknowledgements}

The authors would like to acknowledge useful discussions with Grzegorz Filip and Marc Woolliscroft for assistance in OpenFOAM®. 


\section{Appendix A: Validation Of LES Model And Discretization Schemes}
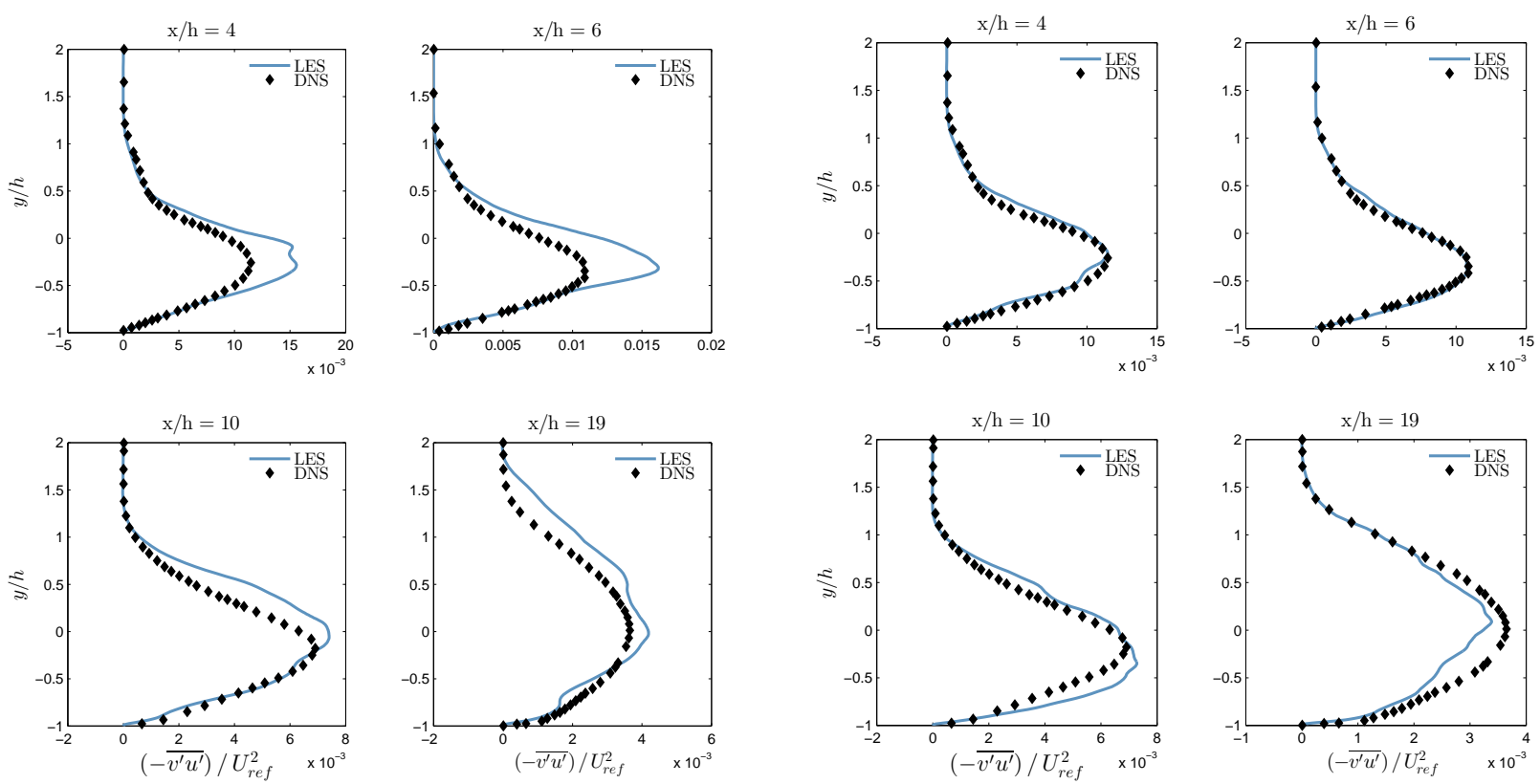

(a) $(-\overline{v / u \prime}) / U_{r e f}^{2}$ for central scheme with explicit correction

(b) $(-\overline{v / u \prime}) / U_{r e f}^{2}$ for central scheme
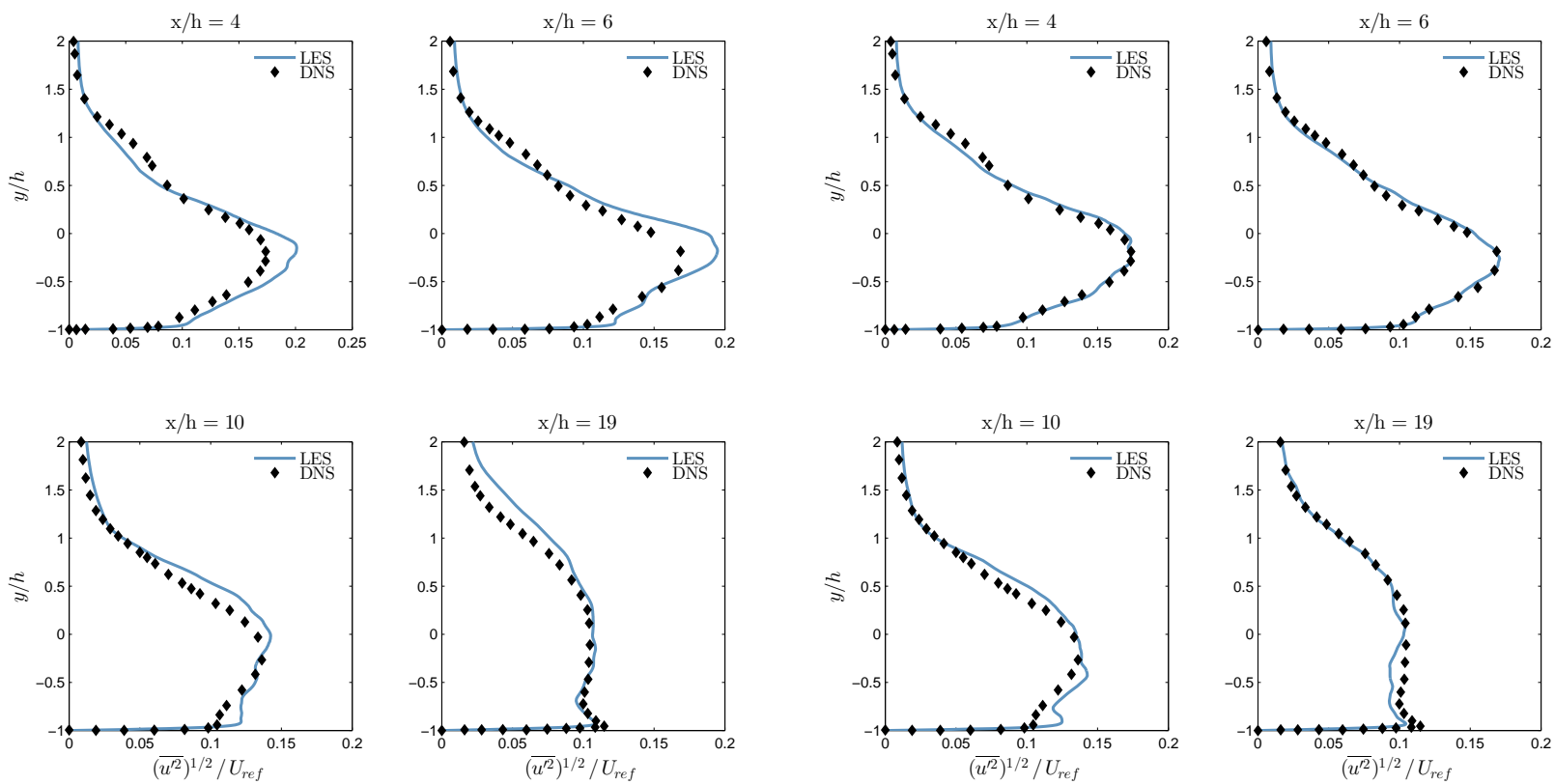

(c) $\left(\overline{u^{\prime}}\right)^{1 / 2} / U_{\text {ref }}$ for central scheme with explicit correction
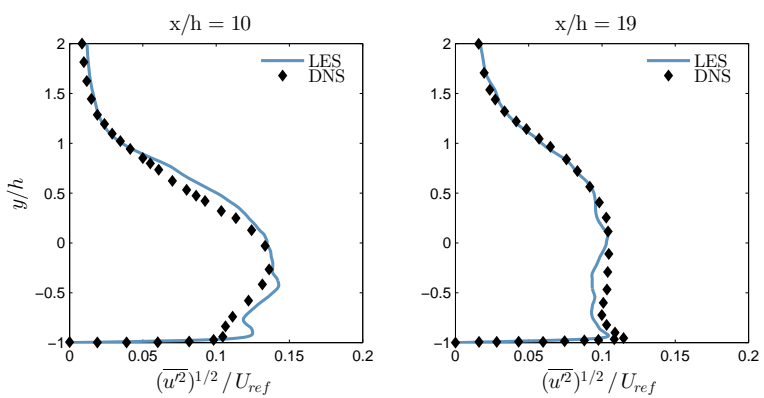

(d) $\left(\overline{u^{\prime}}\right)^{1 / 2} / U_{\text {ref }}$ for central scheme

Figure 8. Comparison of Reynolds stress profiles of backward-facing step simulations with One-Equation Eddy Viscosity LES model and various discretization schemes with DNS data of Le H. et at., $1997 .^{10}$

In order to choose a suitable LES model in OpenFOAM $($ $)$ that predicts the separation with less numerical dissipation, we perform a validation study. A backward-facing step with similar set-up as the DNS study ${ }^{10}$ is simulated with one-equation eddy-viscosity LES model and tested with three different discretization schemes - a linear upwind stabilized scheme, a second-order central scheme and second-order central scheme with explicit correction.

The mean streamwise velocity profiles at different streamwise locations are shown in Figure 9. The mean flow behavior is similar in two of the three discretization schemes as the velocity profiles collapse on each 


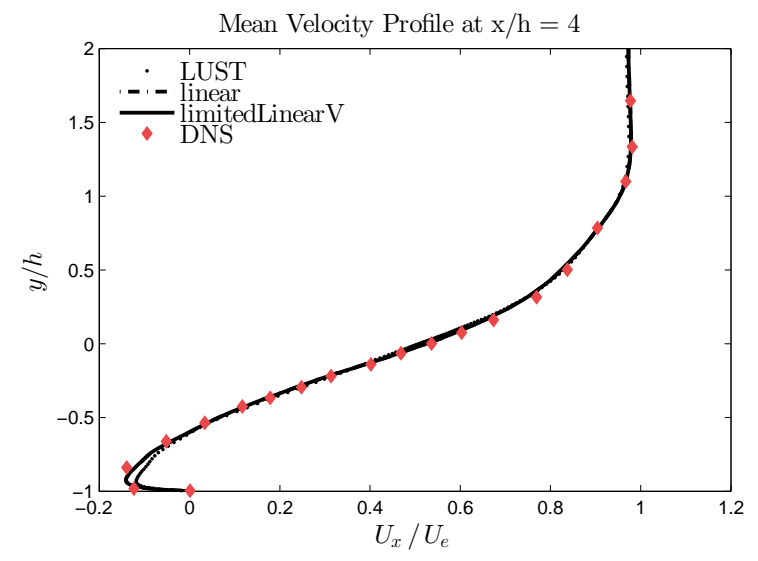

(a) Four $H_{\text {step }}$ after the step.

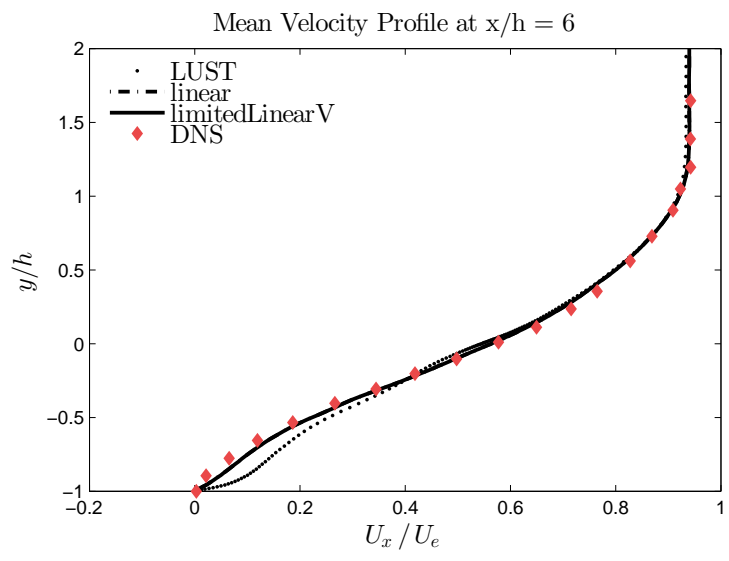

(b) Six $H_{\text {step }}$ after the step.

Figure 9. Streamwise component of the mean velocity profile sampled at different stream wise locations for backwardfacing step simulations with One-Equation Eddy Viscosity LES model and compared with DNS data of Le H. et at., 1997. ${ }^{10}$

other. In order to differentiate, turbulent statistics are shown in Figure 8 for these schemes. We see that the central scheme with one-equation eddy viscosity LES model replicates the flow features and matches DNS data very well. 


\section{Appendix B: Grid Refinement Study}

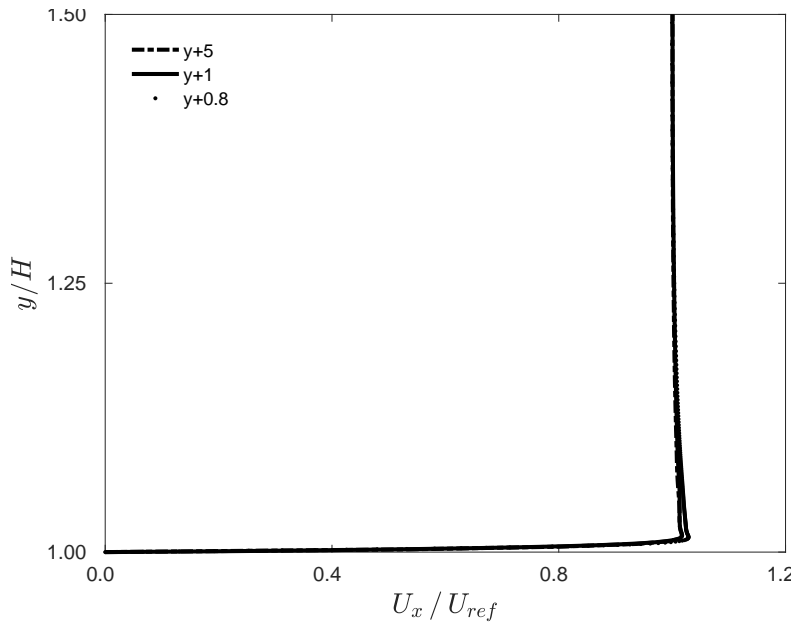

(a) At inlet.

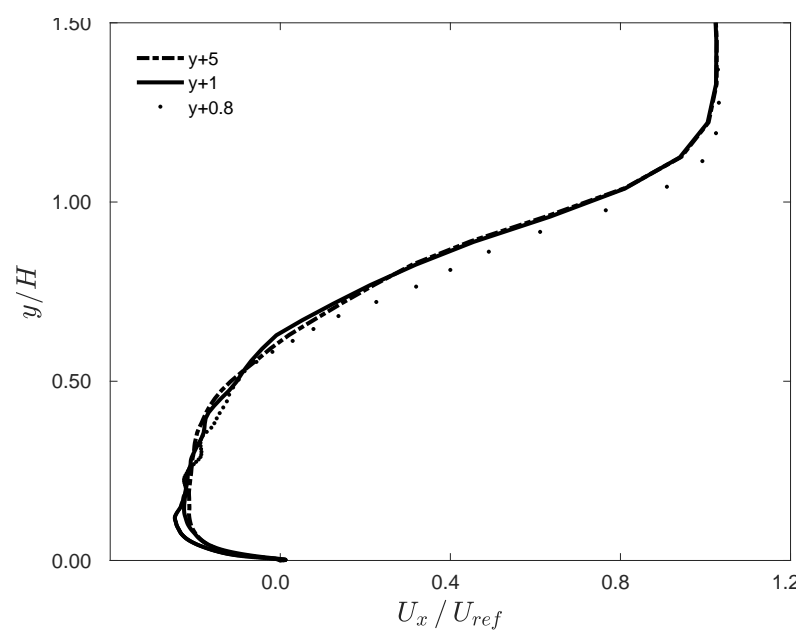

(c) At $x / H=2$.

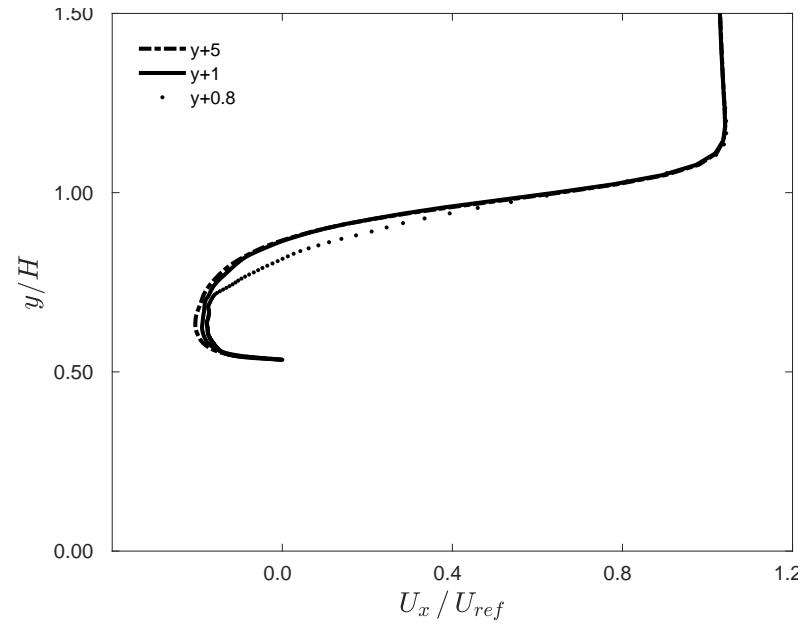

(b) At $x / H=1$.

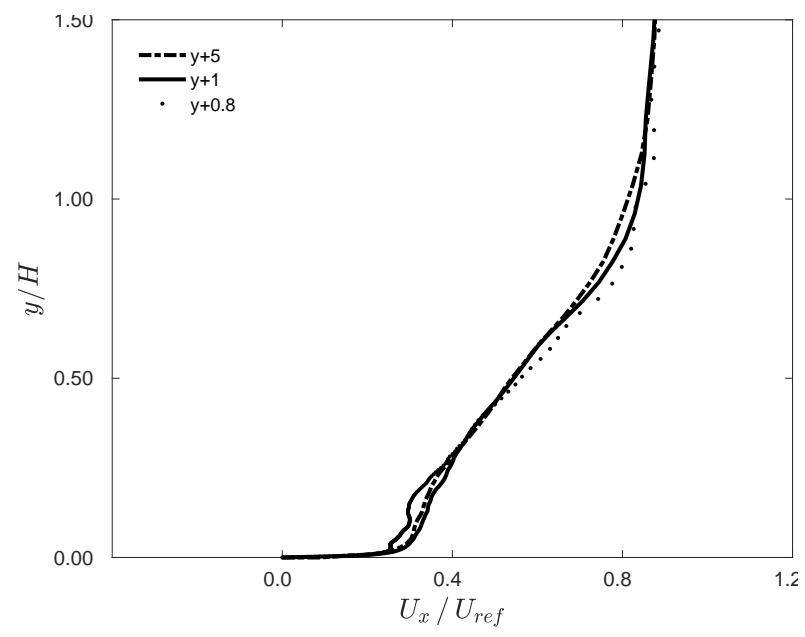

(d) At $x / H=6$.

Figure 10. Streamwise component of the mean velocity profile sampled at different streamwise locations along the center for simulations without a VG on three different grid refinement levels mentioned in Table 2.

It is evident from Figures 10(a)-10(d) that the results with the coarse mesh as mentioned in Table 2 differ slightly from those obtained from the medium and fine meshes. The reason for this disagreement is the inadequate mesh resolution in the coarse mesh simulations near the wall. Especially after the flow separates at the ramp edge the generation of turbulent kinetic energy produces small scale flow structures which cannot be adequately captured by the coarse mesh. Therefore, to obtain mesh independent results it is necessary to have a grid refinement. We observe that the separation region over the ramp surface and the subsequent flow reattachment on the bottom wall is adequately captured by medium and fine grids. Especially the flow behavior near the wall surface is similar. Thus the medium mesh with wall-normal grid spacing of $\Delta_{y}^{+}=1$ is sufficient of our analysis of flow control.

\section{References}

${ }^{1}$ Kerho, M., Hutcherson, S., Blackwelder, R., and Liebeck, R., "Vortex generators used to control laminar separation bubbles," Journal of aircraft, Vol. 30, No. 3, 1993, pp. 315-319.

${ }^{2}$ Brown, A., Nawrocki, H., and Paley, P., "Subsonic diffusers designed integrally with vortex generators." Journal of Aircraft, Vol. 5, No. 3, 1968, pp. 221-229.

${ }^{3}$ Calarese, W. and Crisler, W., "AIM-85-0354 Afterbody Drag Reduction by Vortex Generators," 1985. 
${ }^{4}$ Lin, J. C., "Review of research on low-profile vortex generators to control boundary-layer separation," Progress in Aerospace Sciences, Vol. 38, No. 4, 2002, pp. 389-420.

${ }^{5}$ Rao, D. and Kariya, T., "Boundary-layer submerged vortex generators for separation control-an exploratory study," AIAA paper, Vol. 3546, 1988, pp. 1988.

${ }^{6}$ Krajnovic, S. and Davidson, L., "Large-eddy simulation of the flow around a bluff body," AIAA journal, Vol. 40, No. 5, 2002, pp. 927-936.

${ }^{7}$ Berselli, L. C., T., I., and J., L. W., Mathematics of large eddy simulation of turbulent flows, Scientific Computation, Springer-Verlag, Berlin, 2005.

${ }^{8}$ Germano, M., Piomelli, U., Moin, P., and Cabot, W., "A dynamic subgrid-scale eddy viscosity model," Phys. Fluids, Vol. 3, 1991.

' ${ }^{9}$ Shinde, S., Tandon, S., Johnsen, E., and Maki, K., "Flow separation over a backward-facing ramp with and without a vortex generator," AIAA, 46th Fluid Dynamics Conference, June 2016.

${ }^{10}$ Le, H., Moin, P., and Kim, J., "A direct numerical study of turbulent flow over a backward-facing step," Journal of Fluid Mechanics, Vol. 330, 1997, pp. 349-374.

${ }^{11}$ Hwang, J.-Y. and Yang, K.-S., "Numerical study of vortical structures around a wall-mounted cubic obstacle in channel flow," Physics of Fluids (1994-present), Vol. 16, No. 7, 2004, pp. 2382-2394. 